\title{
Interpolation of spatial temperature profiles by sensor networks
}

\author{
Reiner Jedermann, Javier Palafox-Albarrán \\ Institute for Microsensors, -actors and-systems (IMSAS) \\ University of Bremen \\ Bremen, Germany \\ rjedermann@imsas.uni-bremen.de, \\ Pilar Barreiro, Luis Ruiz-García \\ Polytechnic University of Madrid (UPM), \\ Physical Properties and Advanced Technologies in Agrofood \\ Madrid, Spain
}

\author{
Jose Ignacio Robla \\ National Center for Metallurgical Research, \\ Spanish Council for Scientific Research (CENIM-CSIC), \\ Madrid, Spain
}

Walter Lang

Institute for Microsensors, -actors and-systems (IMSAS)

University of Bremen

Bremen, Germany

\begin{abstract}
The monitoring of spatial profiles of a physical property such as temperature becomes feasible with the decreasing cost of wireless sensor nodes. But to obtain a temperature value for each point in space, it is necessary to interpolate between the existing sensor positions. Accurate spatial temperature supervision is a crucial precondition for maintaining high quality standards in the transportation of food products. The Kriging method was programmed for the ARM processor of the iMote2 sensor nodes and tested with 14 experimental data sets that were recorded in cold storage rooms and transports in trucks and containers. The error of the interpolation by Kriging was $20 \%$ lower than the simpler inverse-distance-weighting used as a reference method.
\end{abstract}

\section{INTRODUCTION}

Keeping the right temperature is the most crucial precondition for improving the quality of fresh fruits during transport and storage. But, this is hindered by local temperature deviations that can be found in almost any truck, container, or storage room. Typical losses along the food chain can sum up to more than $30 \%$ [1]. Equipping each individual packing unit would be the best way to evaluate temperature related quality losses and act accordingly. Despite the decreasing price of sensor nodes, the supervision of transports is still limited to a fixed number of sensors; the temperature for points in-between has to be estimated by a suitable interpolation method.

The aim of this contribution is to test whether the Kriging method [2], which is commonly used in geological sciences, can also be applied within the sensor networks. The two major differences in contrast to a geological setup were considered. A typical setup for monitoring temperature deviations by wireless sensors consists of 20 or 50 nodes, whereas a typical geological setup has several hundred probe points. Furthermore, the data evaluation in geological tasks is done on powerful computers; for the evaluation by a sensor network, the algorithms have to be translated on to small-size embedded microcontrollers.

The accuracy of the Kriging interpolation was tested on 14 experimental data sets, which were recorded in typical food logistic processes. After analysis of these offline data the method was translated to JAVA software, which is fully capable of running on wireless sensor nodes such as the iMote2 [3] equipped with an ARM processor.

Although our test case was limited to local temperature deviations in food logistics, the Kriging method can just as well be applied to interpolate other physical properties measured by sensor networks in other application fields.

\section{APPLIED INTERPOLATION METHOD}

Linear interpolation methods multiply the measured values at the source points with a set of weighting factors in order to estimate the value at a destination point. The weighting factors can be set by a heuristic approach proportional to the inverse squared distance or by-statistically more solid-Kriging method. The Kriging method is based on an analysis of the spatial correlation of the measurements, the so-called Variogram, which gives the expected difference of the physical quantity between two points as a function of their distance. This approach not only provides more accurate interpolation but also a means of calculating the expected interpolation error $\sigma_{\mathrm{k}}$. A detailed introduction to the Kriging method can be found in standard text books [2] or in our previous publication [4].
This research was supported by the German Research Foundation (DFG) as part of the Collaborative Research Centre $637^{\circ}$ 'Autonomous Cooperating Logistic Processes' and by the Federal Ministry of Education and Research, Germany, under reference number 01IA10001 ('The Intelligent Container'). 
The first step to apply the Kriging method comprises of calculating the experimental Variogram from the data set and estimating the parameters of a theoretical model to approximate the experimental function. Typical Variogram models are described by three parameters: range, nugget, and sill. The range gives the maximum distance until which the mutual influence of two probe points has to be considered. Nugget and sill give the expected squared temperature deviation for very small and very large distances.

Two approaches for automated estimation of the Variogram parameters were tested. As the first approach the algorithm from Nelder and Mead [5] was used to minimize the fitting error between the experimental Variogram and the model function. This heuristic algorithm is effective and computationally compact as it does not need any matrix inversion. The fitting error was weighted by a schema according to Cressie [6]. Measurements for small distances/lags are given more weight, but lags with only a small number of available observations are weighted down automatically. The average variance of all measurements $\sigma_{\mathrm{M}}{ }^{2}$ was taken as a raw estimation of the sill value. The search boundaries for the sill were set at $60 \%$ and $180 \%$ of $\sigma_{\mathrm{M}}{ }^{2}$.

Especially the automated estimation of the nugget value turned out to be problematic because our test setup included only few points with small distances. Therefore, a second approach was tested, in which part of the parameters was set to fixed values based on physical considerations or empirical values. The fixed model adapts only the sill value of the individual data set. The range was set by the average of previous experiments under similar conditions. The nugget was directly set to the estimated measurement tolerance of the applied sensor elements. Other factors that could additionally increase the nugget were ignored. The sill was calculated to give the best fit for large distances.

\section{Typical Temperature VARIANCE IN FoOD TRANSPORT AND STORAGE}

The application of the Kriging method was tested on 14 data sets recorded in cold storage rooms, delivery trucks, and

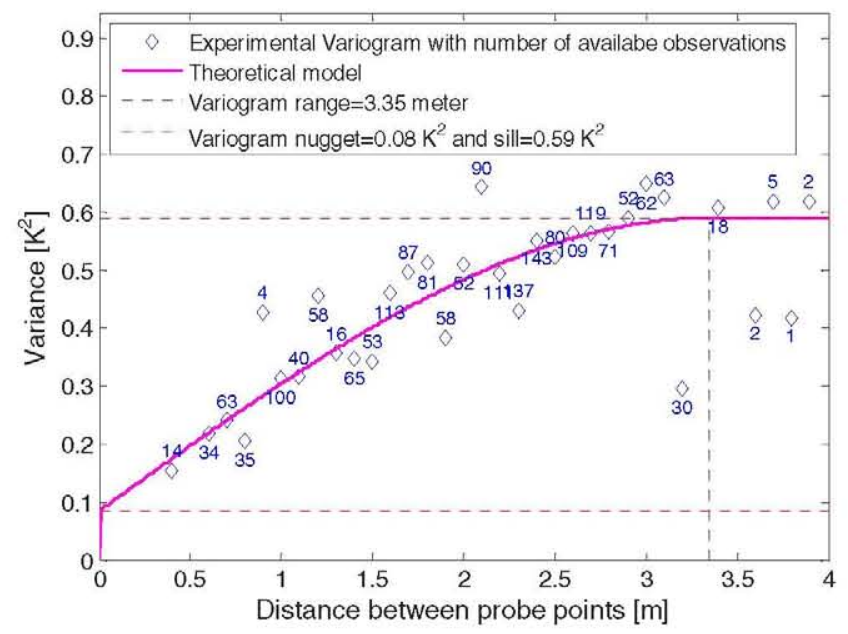

Figure 1. Experimental Variogram for one test in a loaded cold storage room (experiment 8 ) and fitting by a theoretical model. containers. The first 8 sets consist of measurements inside a cold storage room of the size $2.6 \times 2.2 \times 2.3$ meters. The dualstate conditions loading (empty/full), set point $\left(0{ }^{\circ} \mathrm{C} / 6{ }^{\circ} \mathrm{C}\right)$, and type of cooling (on-off /modulated) were altered after each experiment [7]. Between 54 and 68 temperature probes of PT100 type were installed at the walls. In the first step, the Variogram parameters (Fig. 1) were estimated by the NelderMead algorithm. The most significant influence was found for the parameter loading state. After filling the room with water bottles the average range dropped from 4.7 meter to 3.4 meter.

Further data sets were recorded in delivery trucks with 3 separate temperature zones [4] in cooperation with Rungis Express, which is a German food supplier for hotels and restaurants. Two sets (Experiment 9 and 10) were recorded in the deep freezer compartment with a size of $2.9 \times 2.5 \times 2.35$ meters at a set point of $-29^{\circ} \mathrm{C}$. The compartment was partly filled with frozen meat in air-permeable boxes. The manual fitting of the Variogram parameters in [4] was replaced by the automated search described in section 2. The average Variogram range of 3.25 meter was just slightly different from the tests in loaded storage rooms. The Variogram model was used to calculate the weighting factors for interpolation by the Kriging method. Fig. 2 shows an interpolation of the temperature at the walls as an example.

In further tests, the data loggers were not placed at the walls but inside the freight. Two data sets from a sea transport of bananas with 45 sensor positions were provided by Maersk (Experiment 11 and 12). Two sets were recorded within our project in cooperation with Dole on banana transports from Costa Rica to Europe in 2010 and 2011. Twenty-seven and 31 iButton data loggers were packed in the centre of the banana boxes for the experiment 13 and 14, respectively. Because the bananas inside the boxes were packed in a plastic bag to prevent humidity loss, the air could only flow through small gaps between the pallets. This denser packing resulted in a considerable lower Variogram range between 1.13 and 1.65 meter.

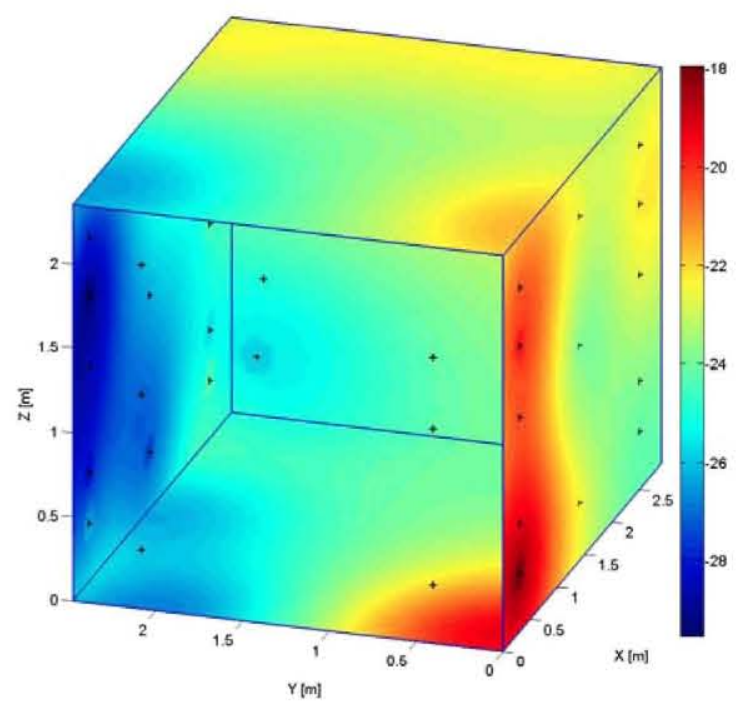

Figure 2. Spatial interpolation of the temperature at the walls of a delivery truck (experiment 10). Crosses mark the sensor positions. 


\section{ACCURACY OF THE INTERPOLATION}

The accuracy of the Kriging interpolation was evaluated by two indicator values. For the first test, only 30 sensors (i.e. source points) of the data sets were taken as input for the interpolation. The temperature for the excluded sensor positions was first interpolated and then the difference to the actual measurements of the excluded positions was calculated. The Root-Mean-Square $\varepsilon$ of the resulting prediction error was taken as the first indicator for the accuracy of the selected interpolation method. The resulting value $\varepsilon$ for Kriging was compared with the inverse-distance-weighting (IDW) and a Null-model as reference. The Null-model ignores any spatial dependency and takes only the average of all sensor measurements as prediction for all points in space (Fig. 3). For the 2 tests in trucks, Kriging is about $70 \%$ better than the Nullmodel. For the 8 tests in the cold storage room, the improvement is about $35 \%$. The improvement compared with the IDW method is in both cases about $20 \%$, but the tests in the container hardly yielded an advantage towards the Nullmodel $(15 \%)$ and none at all compared to IDW.

An internal verification of the Kriging method was used as a second indicator. According to [2] the method can be validated by calculation of the relation between the actual $\varepsilon_{i}$ and the predicted interpolation error $\sigma_{\mathrm{k}}$ per destination point. The Mean-Square of all relation values should be about one. Otherwise, the Variogram model is incorrect, or the expected deviation between two probe points depends not only on their distance, as assumed by Kriging, but also on their absolute positions.

The average relation was slightly closer to 1 for the tests inside a cold storage room when the Variogram parameters were estimated by the Nelder-Mead algorithm (Fig 4), whereas the fixed model clearly gave better results for the truck tests. However, the last container test showed large deviations of the relation for both algorithms.

\section{REQUIRED SENSOR DENSITY}

At first glance, the lower number of sensors in the last two container experiments is the likely reason for their poor

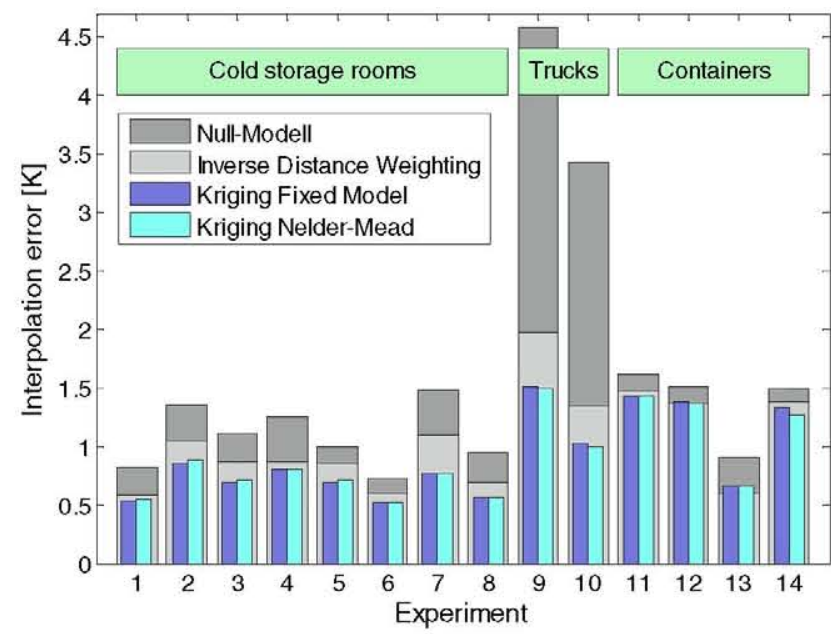

Figure 3. Comparison of the prediction error for different interpolation methods. interpolation results. The number of source points had to be reduced to 20 for the calculation of the indicator values. But a more precise explanation is given, if another factor is considered: the relation between Variogram range and sensors per volume. The container tests have a lower Variogram range and a higher total space in which a similar number of sensors were distributed in common. For the containers, there were only 4 neighboring sensors within the Variogram range for each destination point, whereas the other tests had more than 24 neighbors in range. A further analysis of the experimental data showed that at least 10 neighbors in range are required; otherwise, spatial interpolation is not feasible.

For the truck and cold storage room experiments, it should be questioned, whether it is possible to reduce the number of sensors and thereby save hardware costs without loosing accuracy. A simulation was carried out to estimate the number of required sensors. The simulation started with only 6 sensors placed in the corners of a room. New sensors were added stepby-step at points with the highest expected interpolation error $\sigma_{\mathrm{k}}$. Fig. 5 shows, for example, how the average prediction error $\varepsilon$ decreases with the number of source points for one cold storage room test. But it was not feasible to identify a cut off value for the minimum number of sensors. Their number has to be set in relation to acceptable costs and required accuracy.

\section{EMBEDDED IMPLEMENTATION}

The paramount aim of the project is to extend wireless sensor networks into a cognitive system [8], which evaluates measurements without human interference and decides by itself about necessary actions. The Kriging interpolation was evaluated as one module of such a system. A sensor can, for example, compare its measurements with the interpolation of the neighbors and switch into sleep mode if it detects that it delivers only redundant information. In order to verify that Kriging is suitable for embedded implementation, it was tested to see if the algorithms required for Kriging can be executed on the resource-limited hardware of wireless sensor nodes. Because of the large number of mathematical operations to be performed, the iMote2 sensor nodes from Crossbow [3] with

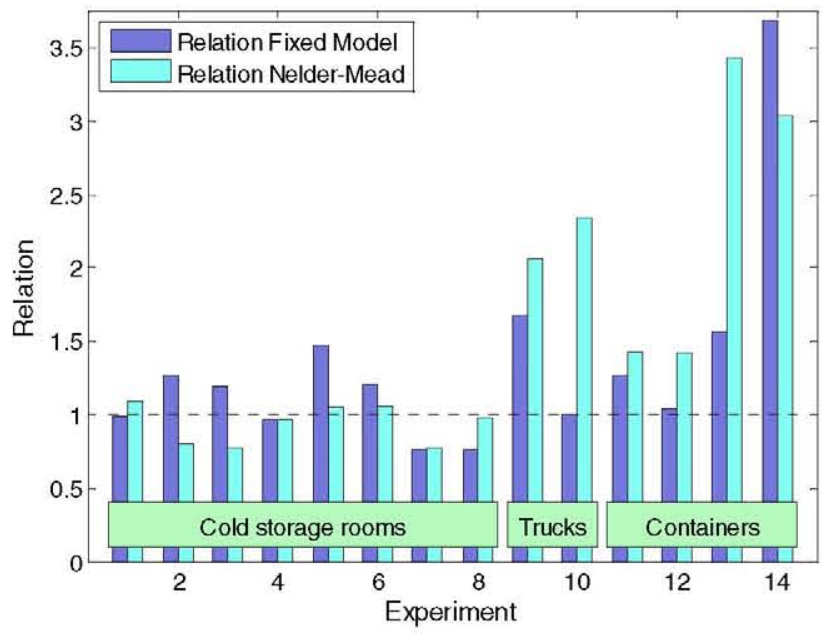

Figure 4. Relation of measured to predicted interpolation error for different experiments 
an ARM PXA271 XScale processor was selected as the test platform. The processor can run at a clock speed of up to 416 $\mathrm{MHz}$, whereas other common sensor node platforms such as the TelosB [9] operate only at $8 \mathrm{MHz}$.

The complete procedure for application of the Kriging method comprises of three sub-algorithms. The Nelder-Mead algorithm was selected for Variogram estimation. As second step the Kriging weights had to be calculated, which required a matrix inversion. The last step was the application of the weights in the form of a matrix multiplication. The related source code was translated from MATLAB to JAVA. The JAVA code was executed by the Jamaica virtual machine [10] that is designed for real-time tasks on embedded systems.

The required CPU time was measured separately for each algorithm. The first two algorithms, which are necessary for the initialization of the interpolation, required a considerable amount of calculation time; but, they are still within the capabilities of the ARM processor. Because they have to be executed only once per transport, the required CPU time of 32 seconds for Nelder-Mead and 2 seconds for matrix inversion, respectively, can be accepted. The application of the Kriging weights subsequent to each measurement took only $17.5 \mathrm{~ms}$.

The execution of complex algorithms on wireless systems is not only a question of the available CPU, but also of the required battery resources. The CPU of the iMote 2 consumes $50 \mathrm{~mA}$ running on full clock speed. But compared with the typical capacity of $950 \mathrm{mAh}$ for a set of AAA batteries, the required energy for Kriging initialization is also not crucial.

On the other hand, the energy balance can be improved, if the number of transmitted messages can be reduced. In order to forward messages in a multi hop sensor network the radio has to be powered up for about 5 seconds with a current consumption of $20 \mathrm{~mA}$ per communication cycle [11]. If one sensor can skip 16 communication cycles, say for example if it has detected that it sends only redundant data, the total energy balance turns to a positive value and the application of Kriging saves more battery energy than it costs.

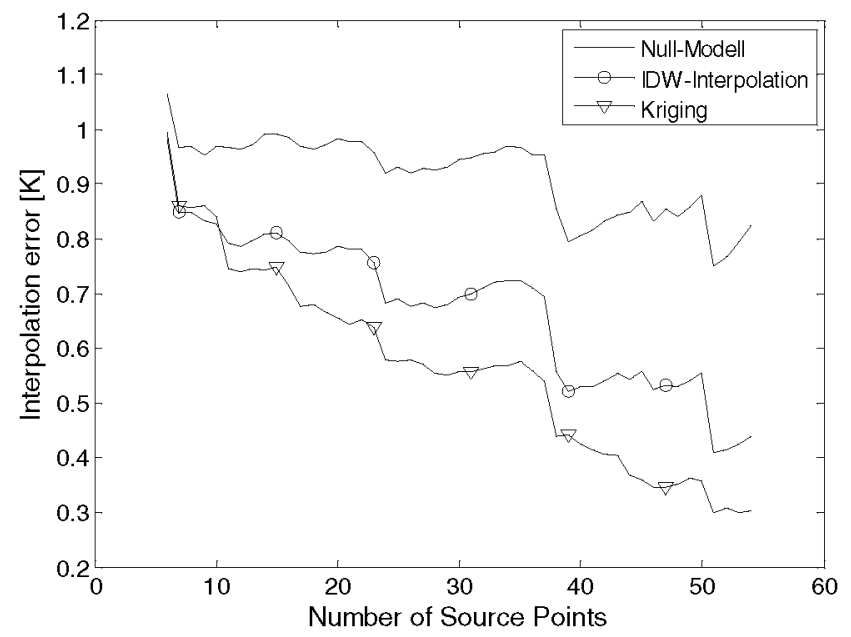

Figure 5. Error as function of number of source points for experiment 8

\section{SUMMARY}

The Kriging method is a useful tool to interpolate a physical property for points where no sensor is available or the sensor is powered down for power saving. Furthermore, Kriging can be used as an offline-tool to estimate to which amount the number of sensors can be reduced for a supervision task. Two different methods were tested for estimation of the Variogram parameters from the experimental data. The most suitable method can be selected based on the $\varepsilon_{\mathrm{i}} / \sigma_{\mathrm{k}}$ relation. If the average number of neighboring sensors within the Variogram range is sufficient, the Kriging method provides an interpolation, which is in average $20 \%$ more accurate than that of the inverse-distance-weighting used as the reference method. The computational recourses that are required by the Kriging method can be met by up-to-date sensor node hardware. The application of spatial interpolation in wireless sensor networks can lead to lower costs by reducing the number of required sensors and extension of the battery life by reduced communication.

\section{ACKNOWLEDGMENT}

R. Jedermann especially thanks Rungis Express Germany, Maersk Copenhagen, and Dole in Costa Rica and Germany for support in field tests and provision of recorded data. Further information about the project can be found at www.intelligentcontainer.com.

\section{REFERENCES}

[1] M. Hülsmann and V. Brenner, "Causes and effects of cold chain ruptures - performance of fragmented versus integrated cold chains", in Contributions to strategic management, ed. M. Hülsmann, Jacobs University Bremen, Bremen, Germany, 2011.

[2] H. Wackernagel, Multivariate geostatistics: An introduction with applications, Springer, 2003.

[3] Crossbow, "Imote2 - high-performance wireless sensor network node", 2007.

[4] R. Jedermann and W. Lang, "The minimum number of sensors interpolation of spatial temperature profiles", Proc. Wireless Sensor Networks, EWSN 2009, Lecture Notes in Computer Science (LNCS), Berlin/Heidelberg, pp. 232-246, 2009.

[5] J. A. Nelder and R. Mead, "A simplex method for function minimization", The Computer Journal, vol.7, no.4, pp. 308-313, 1965.

[6] N. Cressie, "Fitting variogram models by weighted least squares", Mathematical Geology, vol.17, no.5, pp. 563-586, 1985

[7] J. Rodríguez-Bermejo, P. Barreiro, J. I. Robla and L. Ruiz-García, "Thermal study of a transport container", Journal of Food Engineering, vol.80, no.2, pp. 517-527, 2007.

[8] W. Lang, R. Jedermann, D. Mrugala, A. Jabbari, B. Krieg-Brückner and K. Schill, "The intelligent container - a cognitive sensor network for transport management", IEEE Sensors Journal Special Issue on Cognitive Sensor Networks, vol.11, no.3, pp. 688-698, 2011.

[9] Crossbow, "Telosb mote platform", 2005.

[10] F. Siebert, Hard realtime garbage collection, aicas GmbH, Karlsruhe, 2002.

[11] R. Jedermann, M. Becker, C. Görg and W. Lang, "Testing network protocols and signal attenuation in packed food transports", International Journal of Sensor Networks (IJSNet), vol.9, no.3/4, pp $170-181,2011$. 